\title{
Hepatocellular carcinoma: Intratumoral EpCAM-positive cancer stem cell heterogeneity identifies high-risk tumor subtype
}

Jenny Krause ${ }^{1}$, Johann von Felden ${ }^{1}$, Christian Casar ${ }^{2}$, Thorben W. Fründt ${ }^{1}$, Johanna Galaski ${ }^{1}$, Constantin Schmidt ${ }^{1}$, Caroline Jung ${ }^{3}$, Harald Ittrich ${ }^{3}$, Sören A. Weidemann ${ }^{4}$, Till Krech ${ }^{4}$, Asmus Heumann ${ }^{5}$, Jun Li $i^{5}$, Lutz Fischer ${ }^{6}$, Guido Sauter ${ }^{4}$, Ansgar W. Lohse ${ }^{1}$, Henning Wege ${ }^{1}$ and Kornelius Schulze $e^{1,7^{*}}$

\begin{abstract}
Background: The translational interest in the intratumoral heterogeneity of hepatocellular carcinoma (HCC) has been increasing. The dismal prognosis of this pathology is linked to the features of the HCC harbouring cancer stem cells (CSC), represented by EpCAM-expression. However, the extent of the impact of intratumoral distribution of CSC-features, both on the recurrence after curative resection and on clinical outcome, remains unknown. To address this, we investigated the spatial heterogeneity of CSC-features with the aim of identifying the unique HCC patient subgroups amenable to adjuvant treatment.

Methods: We designed a tissue microarray (TMA) from patients who had received liver resection between 2011 and 2017. Tumor specimens were sampled at multiple locations $(n=3-8)$. EpCAM-positivity was assessed for intensity and proportion by applying a score dividing three groups: (i) negative (E-/-); (ii) heterogeneous (E-/+); and (iii) homogeneous $(E+/+)$. The groups were further analysed with regard to time-to-recurrence (TTR) and recurrence-free-survival (RFS).

Results: We included 314 tumor spots from 69 patients (76.8\% male, median age 66, liver cirrhosis/fibrosis 75.8\%). The risk factors were alcohol abuse (26.2\%), NASH (13.1\%), HBV (15.5\%), HCV (17.9\%) and others (27.4\%), representative of a typical Western cohort. E+/+ patients experienced significantly shorter TTR and RFS compared to $\mathrm{E}+/-$ and $\mathrm{E}-/-$ patients (TTR 5 vs. 19 months, $p=0.022$; RFS 5 vs. 14 vs. 21 months, $p=0.016$ ). Only homogeneous EpCAM-positivity correlated with higher AFP levels (> $400 \mathrm{ng} / \mathrm{ml}, p=0.031$ ).

(Continued on next page)
\end{abstract}

\footnotetext{
* Correspondence: k.schulze@uke.de

${ }^{1}$ Department of Medicine, University Medical Centre Hamburg-Eppendorf, Hamburg, Germany

${ }^{7}$ Mildred Scheel Cancer Career Centre HaTriCS4, University Medical Centre Hamburg-Eppendorf, Martinistraße 52, 20246 Hamburg, Germany Full list of author information is available at the end of the article
}

(c) The Author(s). 2020 Open Access This article is licensed under a Creative Commons Attribution 4.0 International License, which permits use, sharing, adaptation, distribution and reproduction in any medium or format, as long as you give appropriate credit to the original author(s) and the source, provide a link to the Creative Commons licence, and indicate if changes were made. The images or other third party material in this article are included in the article's Creative Commons licence, unless indicated otherwise in a credit line to the material. If material is not included in the article's Creative Commons licence and your intended use is not permitted by statutory regulation or exceeds the permitted use, you will need to obtain permission directly from the copyright holder. To view a copy of this licence, visit http://creativecommons.org/licenses/by/4.0/ The Creative Commons Public Domain Dedication waiver (http://creativecommons.org/publicdomain/zero/1.0/) applies to the data made available in this article, unless otherwise stated in a credit line to the data. 
(Continued from previous page)

Conclusions: Spatial heterogeneity of EpCAM-expression was markedly present in the cohort. Of note, only homogeneous EpCAM-expression correlated significantly with early recurrence, whereas heterogeneous EpCAMexpression was associated with clinical endpoints comparable to EpCAM-negativity. We identified a unique HCC subtype associated with a high risk of tumor recurrence.

Keywords: Hepatocellular carcinoma, Cancer stem cell features, EpCAM, Intratumoral heterogeneity

\section{Background}

Hepatocellular carcinoma (HCC) is the most frequent primary liver cancer (90\%) and the 4th leading cause of cancer-related death worldwide [1]. HCC develops in $70-90 \%$ of patients with chronic liver disease, mainly those with liver cirrhosis due to chronic hepatitis B or C, alcohol abuse, non-alcoholic steatohepatitis (NASH), or rare causes such as hemochromatosis. HCC patients are a highly heterogeneous group regarding the diversity of aetiologies and presence or absence of underlying liver disease. This is displayed by intertumoral molecular and histopathological heterogeneity. There is increasing evidence underlining additional significance of intratumoral heterogeneity, as shown by Friemel et al., who demonstrated intratumoral diversity in $87 \%$ of HCC patients by applying morphological and immunohistochemical parameters in a small group of patients [2].

Carcinomas harbouring cancer stem cell (CSC)-features have a dismal prognosis, due to the higher level of invasiveness and greater potential in metastases-dissemination [39]. As a major breakthrough, Yamashita et al. identified EpCAM-positive cancer cell subpopulations in HCC, harbouring the potential for self-renewal, de-differentiation, tumor-initiation, invasiveness, and the capacity to form distant metastases [3, 10, 11]. Moreover, the expression of EpCAM in HCC has been linked to poor prognosis in HCC, suggesting EpCAM as a potential biomarker for risk stratification $[4,11,12]$. However, the degree to which the intensity and spatial distribution of intratumoral EpCAMexpression impacts local aggressiveness and metastasesdissemination remains unclear.

To date, the most effective therapy for HCC is resection at earlier tumor stages, however, tumor recurrence and dismal prognosis are common, because further treatment options are limited to tyrosine kinase inhibitors and palliative care. There is an urgent need to identify risk factors for early metastases-dissemination, local aggressiveness, and recurrence. These risk factors could be decisive for adjuvant treatment in these patients. So far, no adjuvant treatment has proven to be beneficial in HCC, which has been mostly related to the high degree of chemotherapy and radiation resistance of HCC [13].

Therefore, we aimed to determine the spatial heterogeneity of CSC-features by the intensity and proportion of EpCAM-positive cell-populations within different locations of HCC-nodules, and to determine its impact on recurrence and aggressive tumor behaviour. We investigated the clinical impact of intratumoral heterogeneity on outcome, discriminating three pre-defined groups: (i) EpCAM-negative (E-/-); (ii) heterogeneous $(\mathrm{E}-/+)$; and (iii) homogeneous $(\mathrm{E}+/+)$. Finally, we correlated intratumoral EpCAM heterogeneity groups with serum AFP-levels, another surrogate marker for poor prognosis [11], and major clinical endpoints such as TTR and RFS in order to understand whether EpCAMexpression pattern determines HCC subgroups with poor prognosis.

\section{Methods \\ Patients}

For this study, we screened the medical records of 987 patients with a HCC diagnosis, who had been treated at the University Medical Centre Hamburg-Eppendorf between July 2011 and October 2017. Liver resection (LR) was carried out on 69 patients and sufficient tumor tissue was available to prepare a tissue micro-array (TMA). Patients were monitored during the post-operative course and recurrence was diagnosed using crosssectional imaging, as recommended by the guidelines of the European Association for the Study of the Liver (EASL) and the American Association for the Study of Liver Diseases (AASLD) [14, 15]. Where there was inconclusive contrast dynamics, we performed histological confirmation by biopsy. Patients under 18 years of age or who had any active or pre-existing concurrent malignancy were excluded. The study was approved by the Ethics Committee of the Hamburg Medical Association (approval number PV-3578) and all participants provided written informed consent for the study protocol prior to inclusion. Patients were not limited to any type of treatment or line of further treatment. Prior to specific TMA- and immunohistochemistry (IHC)-analysis, tissue samples were reviewed by two independent pathologists from the Institute of Pathology of the University Medical Centre Hamburg-Eppendorf.

\section{Clinical characteristics}

Baseline characteristics such as demographic and other clinical parameters (performance status, risk factors, underlying chronic liver disease, tumor stage according 
to the TNM and BCLC classification), laboratory data (AFP, bilirubin, albumin, and International Normalized Ratio (INR)), and imaging were recorded prior to LR. After LR, the pathological staging was incorporated into the analysis and patients were monitored with regular contrast-enhanced cross-sectional imaging of the liver, clinical examination, and laboratory testing every 3 months in accordance with guideline recommendations $[14,15]$. All patients were monitored until HCC recurrence or effective time of data analysis in July 2019.

\section{Tissue microarray}

A TMA containing 314 tumor spots of the 69 patients was constructed. As controls, we included single tissue spots from non-cancerous liver tissue from HCC patients $(n=31)$ of the cohort described above, and liver tissue from healthy individuals $(n=31)$, and from patients suffering from liver cirrhosis without HCC ( $n=$ 31). Hematoxylin and eosin (H\&E)-stained sections from all paraffin-embedded specimens were reviewed and tumor areas and adjacent liver tissue were marked on the slides. To generate a TMA mapping tumor heterogeneity, 0.6-mm tissue cores were punched out from 3 to 8 different locations of the index area from 1 to 3 different paraffin-embedded tissue specimens, respectively, and transferred into a TMA format as previously described by Kononen [16-18].

\section{Immunohistochemistry}

Freshly cut TMA sections were stained for EpCAM. High-temperature pretreatment of slides was done in a pressure cooker (DAKO buffer, $\mathrm{pH} 6.1, \mathrm{~S} 1699$ ) for 20 min. IHC was performed using a monoclonal antibody (1:10, clone VU-1D9, Novocastra, Newcastle, UK) to detect the membrane-bound positivity for EpCAM protein. The Envision system ${ }^{\circ}$ (DAKO, Glostrup, Denmark) was used for visualization. Staining intensities and proportions of positive tumor cells were analysed for each tissue spot as proposed by the literature. A four-staged scale $(0=$ negative, $1=$ weak, $2=$ moderate and $3=$ strong) was deducted for intensity, and ( $0=$ negative, $1<$ $10 \%, 2=10-50 \%, 3>50 \%$, and $4>75 \%$ positivity) for proportion of positive tumor cells [19]. Fig. 1 displays representative examples of different staining results with regard to proportion and intensity of EpCAMexpression. Due to the size of the nodule, we yielded a different number of spots per tumor. Finally, heterogeneity of EpCAM-expression (intensity and proportion) was assessed by a pre-defined score, differentiating between three groups: (i) EpCAM-negative (E-/-), (ii) heterogeneous $(\mathrm{E}-/+)$, and (iii) homogeneous $(\mathrm{E}+/+)$. We considered both the proportion and intensity of EpCAM-expression within one nodule as major criteria for the classification. In detail, a heterogeneous EpCAM

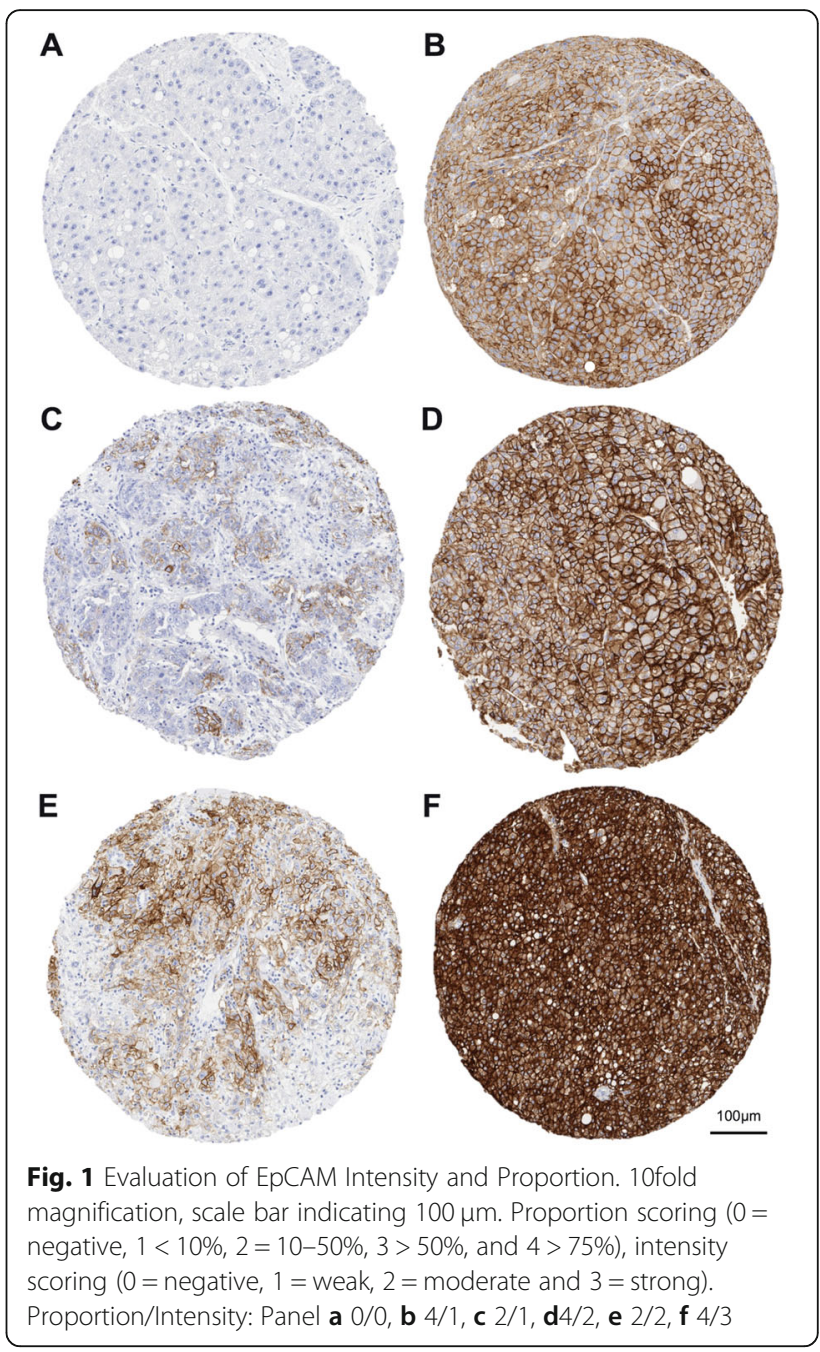

profile $(E-/+)$ is defined if at least one spot is graded 0 and one spot at least 1 for intensity or proportion of EpCAM-expression.

\section{Measurement of serum parameters}

We measured Bilirubin, Albumin and AFP within the routine clinical Dimension Vista ${ }^{\circledR} 1500$ System by Siemens Healthineers.

The INR estimation was based on measurements of the prothrombin time by $\mathrm{BCS}^{\oplus} \mathrm{XP}$ System by Siemens Healthineers.

\section{Statistical analysis}

All analyses were performed using $\mathrm{R}$ (version 3.6, $\mathrm{R}$ Foundation for Statistical Computing). Univariate comparisons of clinical data between pre-defined EpCAMclassification groups were performed using Fisher's Exact test for parametric data and Kruskal-Wallis-test for continuous data. Survival analyses and Cox-Regression modelling was performed with the survival package (version 
2.38). Differences in survival rates at different time points were assessed by administrative censoring of the survival curves at the respective time point and performing a log-rank test implemented in the survdiff function.

We calculated the positive and negative prediction values, and the sensitivity and specificity of a single spot by averaging over 1000 bootstrap samplings of single spots per sample. Classification performance metrics for single spot and all spot prediction of tumor recurrence based on EpCAM intensity and proportion were calculated using the confusionMatrix function provided by the caret package (version 6.0.84). $P$-values below 0.05 were deemed statistically significant.

\section{Results}

Baseline parameters represent a cohort, typical of western countries

Male patients were predominant $[n=53(76.8 \%)]$. The median age was 66 , ranging from 18 to 84 . Of the cases, 39 (63.9\%) were classified BCLC stage A. BCLC stage B $[n=18(29.5 \%)]$ was less frequent, and a few cases with stage $C[n=4(6.6 \%)]$ were included. The majority of patients (98.5\%) were fully active or at least able to carry out work of a light or sedentary nature according to the Eastern Cooperative Oncology Group (ECOG 0 and 1). Chronic liver disease was common as underlying morbidity in our patients. Within our cohort, $41.9 \%$ of patients had liver cirrhosis (Child-Pugh A $88.5 \%$ and B $11.5 \%$ ) and $24.2 \%$ of patients were treated without any histological signs of fibrosis. The remaining patients revealed fibrosis (33.9\%). Known aetiologies for HCC were equally distributed across the cohort with alcohol abuse [22 (26.2\%)], chronic hepatitis C [15 (17.9\%)] and B [13 (15.5\%)], non-alcoholic steatohepatitis (NASH) [11 (13.1\%)], and rare causes or no known underlying risk factor [23 (27.4\%)]. More than two thirds of the patients (73\%) underwent resection for a single lesion; only a minority presented 4 or more HCC-nodules (11.1\%). Pathological reviewing classified $76.8 \%$ of patients T1 or T2, 98.5\% exhibited N0, 98.5\% L0, and 91.3\% R0. 62.3\% of patients revealed neither microscopic nor macroscopic vascular invasion. The majority (71\%) of tumors were moderately differentiated (G2), 15.9\% well differentiated (G1), and $13 \%$ poorly differentiated (G3). Overall, our patients represented a typical cohort undergoing curative resection in Western countries. Clinical baseline and general histopathological tumor characteristics are summarized in Table 1.

Spatial heterogeneity of EpCAM was markedly present

We confirmed the presence of spatial heterogeneity of CSC-features, measured by intensity and proportion of EpCAM-expression within the same tumor nodule. Twenty-nine patients (42\%) did not exhibit any EpCAM- expression (E-/-), whereas 7 patients (10.1\%) stood out with homogeneous EpCAM-expression throughout all tumor spots $(\mathrm{E}+/+)$. Thirty-three patients $(47.8 \%)$ contained tumor spots with and without EpCAMexpression of diverse quality, confirming the presence of spatial heterogeneity of CSC-features in nearly half of patients with HCC (E-/+) (Fig. 2a). EpCAM expression of control tissues of chronic liver diseases did not correlate with aetiology or HCC recurrence (Fig. 2b). Figure 2 illustrates the results of EpCAM-staining in intensity and proportion in all 314 tumor spots with regard to EpCAM-classification score [negative (E-/-), heterogeneous $(\mathrm{E}-/+)$, homogeneous $(\mathrm{E}+/+)]$ and in correlation with baseline features.

\section{Only homogeneous EpCAM-expression correlated significantly with early recurrence}

Based upon the EpCAM-score of the groups [(i) negative (E-/-), (ii) heterogeneous (E-/+), (iii) homogeneous $(\mathrm{E}+/+)]$, we were interested in the outcome of the patients in order to understand whether EpCAMexpression determined a tumor subgroup characterized by poor prognosis.

First, we checked for group comparability with regard to the baseline characteristics (Table 2). We did not find any significant differences in the demographic data, performance status, risk factors, presence or absence of underlying chronic liver disease, liver function (bilirubin, albumin, INR estimation), or staging according to the BCLC classification between the EpCAM-classification groups. Likewise, there were no significant differences between the three groups with regard to the $\mathrm{L}_{-}, \mathrm{V}-, \mathrm{N}-$, R- or G-stage. Overall, we did not identify any confounders between the three groups for our final outcome analysis.

To test for the clinical impact of EpCAM expression in HCC, we determined the TTR and the RFS for early recurrence (i.e. within 24 months). Recurrence within the first 24 months after curative resection is considered more likely to be true recurrence instead of de novo tumors. As highlighted in Fig. 3, TTR is significantly shorter at 5 months in patients with $\mathrm{E}+/+$ compared to patients with $\mathrm{E}+/-$ at 19 months $(p=0.022)$. The median TTR was not reached for patients scored E-/-. The hazard ratio for recurrence between $\mathrm{E}+/+$ and $\mathrm{E}-/$ - was 3.8 (95\% CI, 1.32-11.2, $p=0.014$ ). Results for RFS, depicted in Fig. 4, were also significantly different at 5 months for $\mathrm{E}+/+$ compared to 14 months for E-/+, and 21 for E-/$(p=0.016)$. The hazard ratio was 3.6 (95\% CI, 1.39-9.5, $p=0.009$ ). Interestingly, in both endpoints, TTR and RFS, we found a similar outcome in $\mathrm{E}+/-$ and $\mathrm{E}-/$ groups (Figs. 3 and 4). Recurrence-free survival rates at 24 months were 57.2 and $35.6 \%$ for the $E-/-$ and $E+/-$ groups $(\mathrm{p}=\mathrm{n} . \mathrm{s}$.$) , respectively. No patient in the \mathrm{E}+/+$ 
Table 1 Clinical variables for the overall cohort. Aetiology variable may include multiples risk factors

\begin{tabular}{|c|c|}
\hline Baseline Characteristics & Overall $n=$ \\
\hline \multicolumn{2}{|l|}{ Sex } \\
\hline m & $53(76.81)$ \\
\hline f & $16(23.19)$ \\
\hline \multicolumn{2}{|c|}{ Age at surgical procedure (years) } \\
\hline Range & $18-84$ \\
\hline Median (n) & $66(69)$ \\
\hline \multicolumn{2}{|l|}{$\mathrm{ECOG}(n=67)$} \\
\hline 0 & $37(55.22)$ \\
\hline 1 & $29(43.28)$ \\
\hline 2 & $1(1.49)$ \\
\hline \multicolumn{2}{|l|}{$\mathrm{BCLC}(n=61)$} \\
\hline A & $39(63.93)$ \\
\hline B & $18(29.51)$ \\
\hline C & $4(6.56)$ \\
\hline \multicolumn{2}{|c|}{ Histological Stage of Liver $(n=62)$} \\
\hline normal & $15(24.19)$ \\
\hline Fibrosis & $21(33.87)$ \\
\hline Cirrhosis & $26(41.94)$ \\
\hline \multicolumn{2}{|l|}{ Child-Pugh Stage $(n=26)$} \\
\hline A & $23(88.46)$ \\
\hline B & $3(11.54)$ \\
\hline \multicolumn{2}{|l|}{ Aetiology } \\
\hline Alcohol & $22(26.19)$ \\
\hline HBV & $13(15.48)$ \\
\hline $\mathrm{HCV}$ & $15(17.86)$ \\
\hline NASH & $11(13.1)$ \\
\hline Other & $9(10.71)$ \\
\hline w/o liver disease & $14(16.67)$ \\
\hline \multicolumn{2}{|c|}{ Number of HCC-lesions ( $n=63$ ) } \\
\hline 1 & $46(73.02)$ \\
\hline 2 & $5(7.94)$ \\
\hline 3 & $5(7.94)$ \\
\hline$>=4$ & $7(11.11)$ \\
\hline \multicolumn{2}{|l|}{ T Stage $(n=69)$} \\
\hline $\mathrm{T} 1$ & $28(40.58)$ \\
\hline $\mathrm{T} 2$ & $25(36.23)$ \\
\hline T3 & 16 (23.19) \\
\hline \multicolumn{2}{|l|}{ N Stage $(n=69)$} \\
\hline No & $68(98.55)$ \\
\hline N1 & $1(1.45)$ \\
\hline \multicolumn{2}{|l|}{ L Stage $(n=69)$} \\
\hline LO & $68(98.55)$ \\
\hline L1 & $1(1.45)$ \\
\hline
\end{tabular}

Table 1 Clinical variables for the overall cohort. Aetiology variable may include multiples risk factors (Continued)

\begin{tabular}{ll}
\hline Baseline Characteristics & Overall $\boldsymbol{n}=\mathbf{6 9}(\%)$ \\
\hline V Stage $(n=69)$ & $43(62.32)$ \\
V0 & $26(37.68)$ \\
V1 & \\
R Stage $(n=69)$ & $63(91.3)$ \\
R0 & $6(8.7)$ \\
R1 & \\
G Stage $(n=69)$ & $11(15.94)$ \\
G1 & $49(71.01)$ \\
G2 & $9(13.04)$ \\
G3 &
\end{tabular}

group remained without recurrence or death in the 24 months after resection. In summary, we demonstrated a significantly worse prognosis for patients with homogeneous EpCAM-expression, and also showed that heterogeneous EpCAM and negative EpCAM-expressing tumors exhibited a similar outcome, highlighting the clinical impact of thorough assessment of spatial EpCAM-expression in HCC nodules.

\section{Homogeneous EpCAM-expression was indicative for local aggressiveness and tumor dissemination}

We observed that the group of $\mathrm{E}+/+$ patients was distinct from $\mathrm{E}+/-$ and $\mathrm{E}-/-$ patients in the context of tumor aggressiveness (serum AFP-levels $(</ \geq 400 \mathrm{ng} / \mathrm{mL}$ ) and number of HCC nodules). Remarkably, $\mathrm{E}+/-$ and $\mathrm{E}$ -/- demonstrated not only similar clinical behaviour, but also similar serum AFP-levels, and number of HCC nodules. So we wondered whether these surrogate parameters also statistically discriminated the group of homogeneous EpCAM-expressing HCC from the negative and heterogeneous expressing tumors, as described for EpCAM-expressing tumors in general by Yamashita et al. [11] Therefore, we assessed AFP serum levels prior to resection, as well as the number of satellite lesions. In HCC patients, an AFP concentration higher than 400 $\mathrm{ng} / \mathrm{mL}$ has been consistently associated with poor prognosis in several treatment settings and used as the cutoff for several clinical trials such as REACH and REAC $\mathrm{H}-2$ [20]. AFP levels were significantly higher in the $\mathrm{E}+/+$ group compared to the other groups. In detail, $57 \%$ of patients with $\mathrm{E}+/+$ presented AFP levels $>400$ $\mathrm{ng} / \mathrm{mL}$ compared to $13.8 \%$ in $\mathrm{E}+/-$ and $11.5 \%$ in $\mathrm{E}-/-$ ) $(p=0.031)$. Correspondingly, patients with $\mathrm{E}-/-$ and $\mathrm{E}+/$ - status harboured significantly less satellite tumor lesions at the time of resection compared to the $\mathrm{E}+/+$ group $(p=0.006)$ (Table 3.)

In summary, homogeneous EpCAM-expression was significantly indicative for higher local aggressiveness 


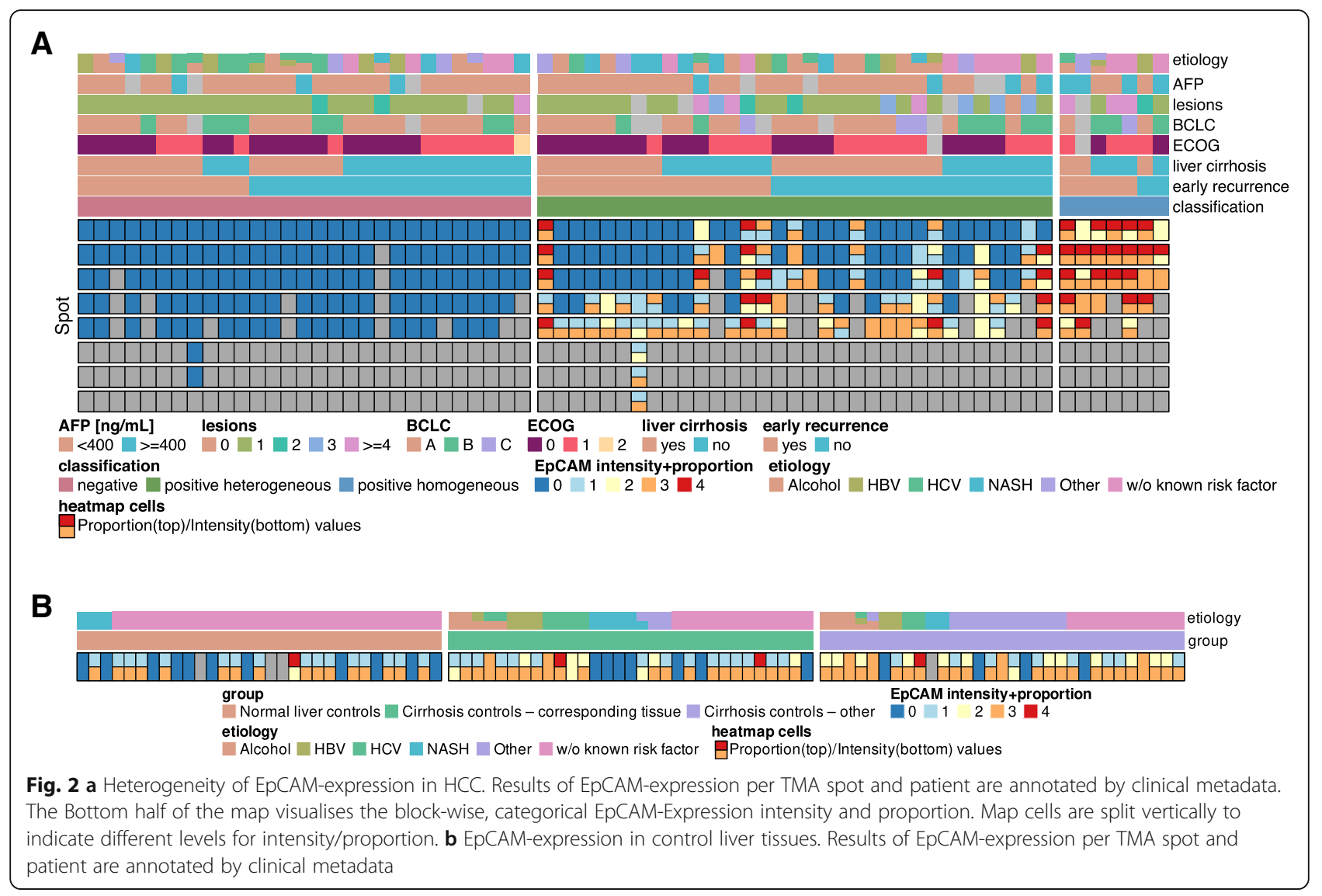

and earlier tumor dissemination compared to heterogeneous EpCAM-expression. EpCAM-negative tumor nodules demonstrated similar low aggressiveness in tumor features like EpCAM-heterogeneous tumor nodules.

\section{The positive predictive value of a single biopsy to diagnose} HCC with homogeneous EpCAM-expression was only $45 \%$ Since only homogeneous EpCAM-expression was associated with more aggressive tumor behaviour and subsequently, a poorer outcome, we were interested in the predictive value of a single biopsy towards the overall EpCAM-score (E+/+, E+/-, or E-/-). Therefore, we assessed the positive and negative prediction values, and the sensitivity and specificity of the EpCAM expression pattern of a single spot. We discovered that the sensitivity for a single spot was $100 \%$, the specificity was $87 \%$, the negative predictive value was $100 \%$, but the positive predictive value was only $45 \%$. Moreover, the positive predictive value of a single spot for early recurrence within 24 months after resection was $58 \%$, the negative predictive value was $58.5 \%$, the sensitivity was $27 \%$, and the specificity was $84 \%$.

\section{Discussion}

Personalized medicine and clinical decision-making have been seeing an increase in the phenotypic and histopathological characterization of malignancies. In HCC, however, adjuvant treatment in patients has not yet been established because of the lack of positive phase 3 clinical trials, likely due to insufficient patient selection in recent studies. It is believed that certain patients could benefit from approved treatment modalities, e.g. transarterial chemoembolization (TACE), tyrosine kinase inhibitors, and immune checkpoint inhibitors, in an adjuvant setting under consideration of stringent selection criteria. Similar to colorectal cancer, high or low risk features for early metastasesdissemination and local aggressiveness could be key for adjuvant treatment after curative-intended resection. For this reason, CSC-features, in particular EpCAM-expression, are a highly promising surrogate marker in HCC identification of patient groups at risk. In addition, stratification on the basis of the underlying tumor subtype is urgently required in HCC patients. However, the extent to which intratumoral heterogeneity of EpCAM expression is present in HCC nodules, and how it might affect clinical outcome of patients has never before been evaluated.

To our knowledge, our study is the most extensive one of its kind to investigate the spatial CSC heterogeneity in HCC. We used it to verify that EpCAM-expression was heterogeneously distributed within tumor nodules. Homogeneous EpCAM-expression was present in only 1 
Table 2 Cohort split into EpCAM classification groups. Group differences assessed by Fisher's Exact test for categorical variables and Kruskal-Wallis-test for numerical variables

\begin{tabular}{|c|c|c|c|c|}
\hline $\begin{array}{l}\text { Baseline } \\
\text { Characteristics }\end{array}$ & $\begin{array}{l}\text { EpCAM-negative } n=29 \\
(42.1 \%)\end{array}$ & $\begin{array}{l}\text { EpCAM- heterogeneous } n=33 \\
(47.8 \%)\end{array}$ & $\begin{array}{l}\text { EpCAM- homogeneous } n=7 \\
(10.1 \%)\end{array}$ & $\begin{array}{l}p- \\
\text { value }\end{array}$ \\
\hline \multicolumn{5}{|l|}{ Sex } \\
\hline m & $24(77.42)$ & $26(78.79)$ & $5(71.43)$ & \multirow[t]{2}{*}{0.920} \\
\hline f & $7(22.58)$ & $7(21.21)$ & $2(28.57)$ & \\
\hline \multicolumn{5}{|c|}{ Age at surgical procedure (years) } \\
\hline range & $38-84$ & $18-81$ & $58-76$ & \multirow[t]{2}{*}{0.326} \\
\hline median (n) & $69(29)$ & $66(33)$ & $63(7)$ & \\
\hline \multicolumn{5}{|l|}{ ECOG } \\
\hline 0 & $17(58.62)$ & $18(56.25)$ & $2(33.33)$ & \multirow[t]{3}{*}{0.535} \\
\hline 1 & $11(37.93)$ & $14(43.75)$ & $4(66.67)$ & \\
\hline 2 & $1(3.45)$ & $0(0)$ & $0(0)$ & \\
\hline \multicolumn{5}{|l|}{ BCLC } \\
\hline A & $19(70.37)$ & $18(64.29)$ & $2(33.33)$ & \multirow[t]{3}{*}{0.152} \\
\hline B & $8(29.63)$ & $7(25)$ & $3(50)$ & \\
\hline C & $0(0)$ & $3(10.71)$ & $1(16.67)$ & \\
\hline \multicolumn{5}{|c|}{ Histological Stage of Liver } \\
\hline No Fibrosis & $6(22.22)$ & $6(21.43)$ & $3(42.86)$ & \multirow[t]{3}{*}{0.808} \\
\hline Fibrosis & $10(37.04)$ & $9(32.14)$ & $2(28.57)$ & \\
\hline Cirrhosis & $11(40.74)$ & $13(46.43)$ & $2(28.57)$ & \\
\hline \multicolumn{5}{|l|}{ Child Pugh Stage } \\
\hline A & $11(100)$ & $11(84.62)$ & $1(50)$ & \multirow[t]{2}{*}{0.111} \\
\hline B & $0(0)$ & $2(15.38)$ & $1(50)$ & \\
\hline \multicolumn{5}{|l|}{ Aetiology } \\
\hline Alcohol & $9(24.32)$ & $11(29.73)$ & $2(20)$ & \multirow[t]{6}{*}{0.617} \\
\hline HBV & 7 (18.92) & $4(10.81)$ & $2(20)$ & \\
\hline $\mathrm{HCV}$ & $9(24.32)$ & $5(13.51)$ & $1(10)$ & \\
\hline NASH & $4(10.81)$ & $7(18.92)$ & $0(0)$ & \\
\hline Other & $4(10.81)$ & $3(8.11)$ & $2(20)$ & \\
\hline w/o liver disease & $4(10.81)$ & 7 (18.92) & $3(30)$ & \\
\hline \multicolumn{5}{|l|}{ Bilirubin } \\
\hline range & $0.3-1.7$ & $0.3-2.3$ & $0.4-0.7$ & \multirow[t]{2}{*}{0.105} \\
\hline median (n) & $0.65(22)$ & $0.5(25)$ & $0.5(7)$ & \\
\hline \multicolumn{5}{|l|}{ Albumin } \\
\hline range & $31-43$ & $2.34-43$ & $30-42$ & \multirow[t]{2}{*}{0.152} \\
\hline median (n) & $38(20)$ & $36(21)$ & $35(6)$ & \\
\hline \multicolumn{5}{|l|}{ INR } \\
\hline range & $0.93-2.42$ & $0.92-3.08$ & $0.95-1.25$ & \multirow[t]{2}{*}{0.863} \\
\hline median (n) & $1.06(25)$ & $1.055(24)$ & $1.06(6)$ & \\
\hline
\end{tabular}

out of 10 patients, whereas EpCAM heterogeneity was found in every other patient. EpCAM-expression of chronic liver disease control tissues did not correlate with aetiology or HCC recurrence (Fig. 2b). Homogeneous distribution of EpCAM-expression led to earlier dissemination, recurrence and/or death after curative-intended resection when compared with heterogeneous expressing tumors.

When assessing clinical baseline characteristics, we did not observe any differences in risk factors between the groups. Importantly, group determination was not 


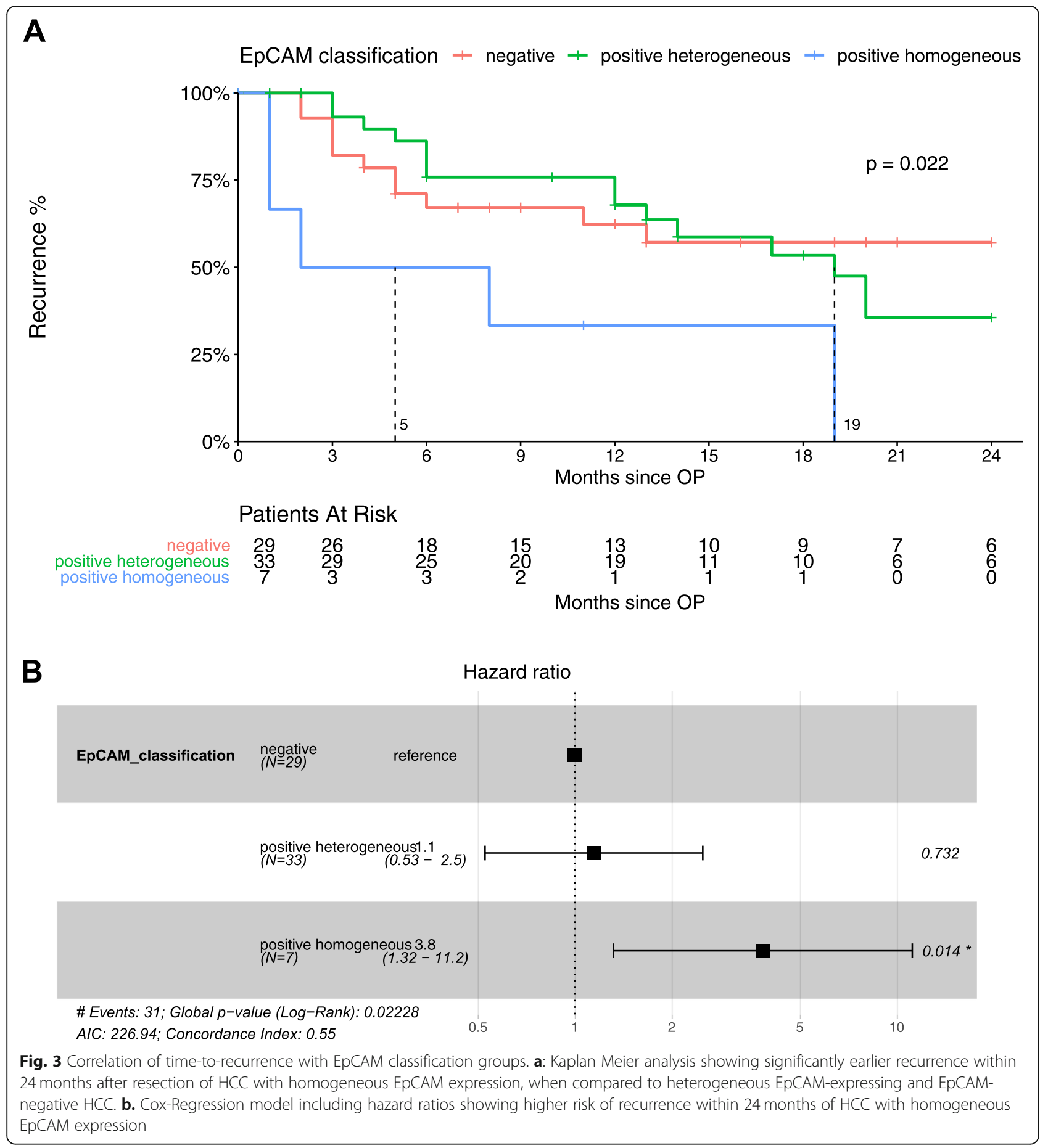

influenced by the tumor status at the time of resection (BCLC), by the parameters of liver function such as bilirubin, albumin and INR estimation, or by the aetiology of the underlying liver disease. However, homogeneous distribution of EpCAM-expression was significantly associated with higher serum AFP-levels $(p=0.03)$ and the number of intrahepatic satellite HCC lesions $(p=0.006)$ compared with heterogeneous EpCAM expression, confirming former studies of Bae et al. and Yamashita et al. [11, 21] Of note, all E+/- and E-/- cases had similar clinical outcomes, suggesting the same biological behaviour in HCC with none or low expression pattern of CSC features. In short, our findings identified a new subgroup of HCC patients at high risk of early recurrence and death.

Hepatic stem and progenitor cells can be the source of tumor initiation and CSC can drive hepatocarcinogenesis 


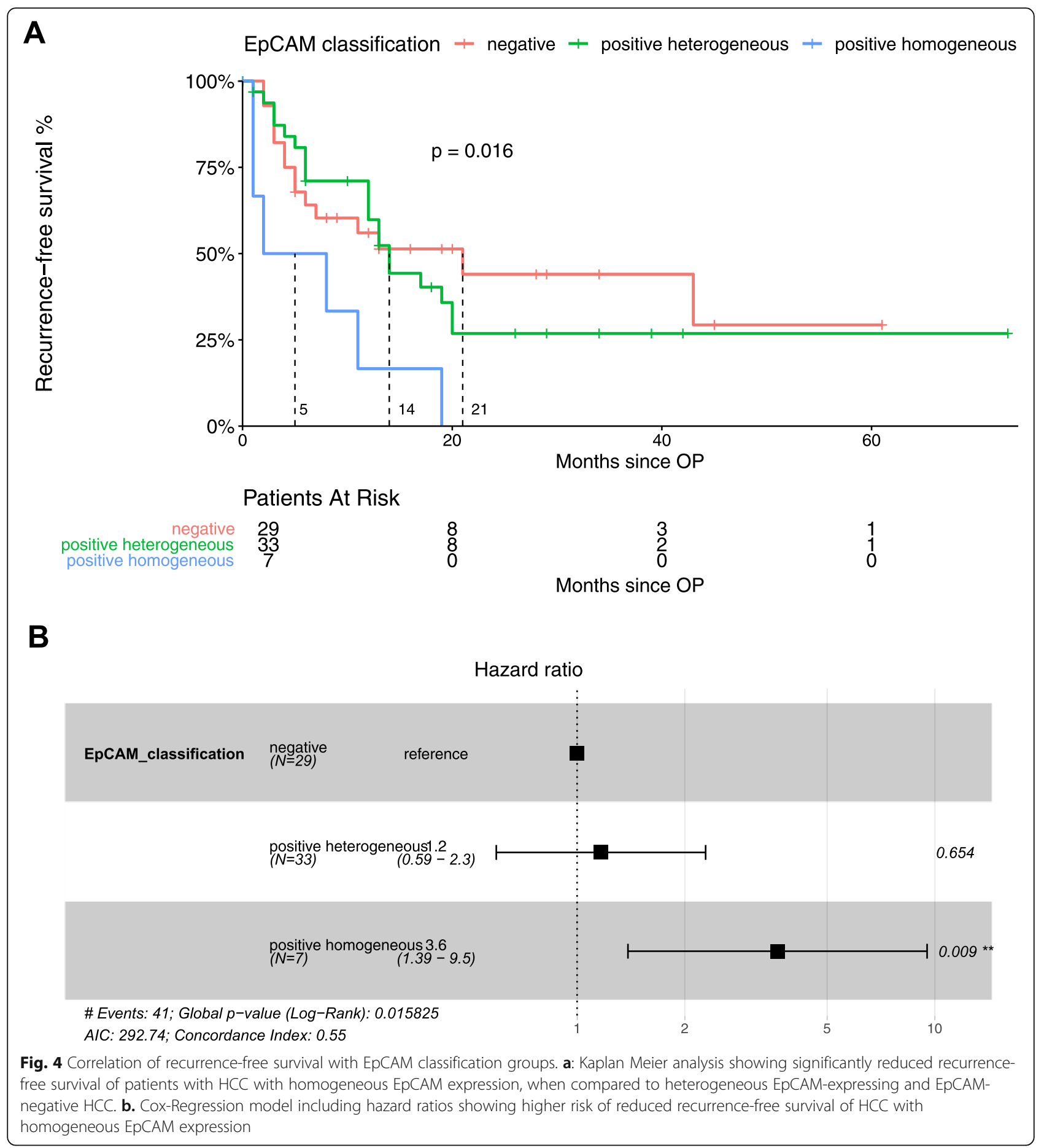

[22, 23]. Moreover, EpCAM expression is linked to CSC features in various forms of cancer such as gastric, prostate, pancreatic and breast. What's more, it is linked to a dismal prognosis and to chemotherapy resistance [6-9]. Our study highlights that only a full-scale CSC milieu within HCC nodules was of clinical significance in terms of prognosis, affirming and specifying previous findings [4, 11, 24].
Substantial EpCAM-expression is known to be the result of a distinct underlying molecular and mutational profile as introduced by molecular classifications [25]. Further studies investigating the transcriptional characterization of EpCAM-expressing tumor cells are required. $\mathrm{HCC}$ can be categorized into proliferation and non-proliferation classes. EpCAM positivity and abundant CSC features are associated with the G1/S2/ 
Table 3 Surrogate markers for local aggressiveness, de-differentiation, tumor-initiation, and invasiveness in patients with HCC, considering EpCAM classification groups. Group differences assessed by Fisher's Exact test for categorical variables and Kruskal-Wallistest for numerical variables

\begin{tabular}{|c|c|c|c|c|}
\hline $\begin{array}{l}\text { Surrogate marker for CSC- } \\
\text { features }\end{array}$ & $\begin{array}{l}\text { EpCAM-negative } n=29 \\
(42.1 \%)\end{array}$ & $\begin{array}{l}\text { EpCAM- heterogeneous } n=33 \\
(47.8 \%)\end{array}$ & $\begin{array}{l}\text { EpCAM- homogeneous } n=7 \\
(10.1 \%)\end{array}$ & $\begin{array}{l}p \text { - } \\
\text { value }\end{array}$ \\
\hline \multicolumn{5}{|l|}{ Number of HCC nodules } \\
\hline 1 & $25(89.29)$ & $19(65.52)$ & $2(33.33)$ & \multirow[t]{4}{*}{0.006} \\
\hline 2 & $2(7.14)$ & $2(6.9)$ & $1(16.67)$ & \\
\hline 3 & $0(0)$ & $5(17.24)$ & $0(0)$ & \\
\hline$\geq 4$ & $1(3.57)$ & $3(10.34)$ & $3(50)$ & \\
\hline \multicolumn{5}{|l|}{ Serum AFP-levels } \\
\hline$<400$ & $23(88.46)$ & $25(86.21)$ & $3(42.86)$ & \multirow[t]{2}{*}{0.031} \\
\hline$\geq 400$ & $3(11.54)$ & $4(13.79)$ & $4(57.14)$ & \\
\hline
\end{tabular}

iCluster 1 , termed the progenitor group. This group is characterized by RPS6KA3, TP53, and AXIN1 mutations and by IGF1R, AKT/mTOR signalling [25]. Thus, it will be crucial to identify the underlying mutational profile, eventually leading to $\mathrm{HCC}$ with homogeneous or heterogeneous EpCAM distribution. WNT-ß-catenin activation via transcription factor TCF-4 is also associated with transcriptional activation of EpCAM expression [26]. A Japanese study demonstrated in two human HCC samples inactivating TP53 mutations (c.844C > T; c.767C > $\mathrm{T})$ in EpCAM positive HCC [23]. In HCC, activating CTNNB1 mutations and inactivating TP53 mutations are major oncogenic events with frequencies of up to 37 and $24 \%$, respectively $[27,28]$. They could in part be reasonable drivers in homogeneous distribution in EpCAM expression. Due to methodological limitations of the present study, analysis of the mutational background was not within the scope of this work, but future studies will need to address the molecular and genetic impact on homogeneous or heterogeneous CSC patterns. Ultimately, it will be necessary to study CSC heterogeneity within a larger patient cohort at a clonal level through single cell analysis. The impact will need to be confirmed by functional experiments. In a proof of concept study, Ho et al. performed scRNA-sequencing on dissociated HCC tumor cells, and observed two subpopulations distinguished mainly by the expression of EpCAM and stemness-related genes, underlining the significance of this finding, even though the study was limited to a single specimen [29].

Our comprehensive data allowed us to demonstrate that clinical parameters, mainly risk factors and presence of underlying chronic liver disease, are not involved in determining the fate of CSC expression pattern in HCC.

We believe that histopathological investigation of resected HCC lesions is key to stratify patient management. With regard to intensity and proportion of CSC features, a simple EpCAM score could estimate the risk of recurrence. Subsequently, the score could be used to establish a clinical trial to investigate potential adjuvant treatment options where patients could be stratified in groups using the score proposed in this study.

Several trials in HCC, predominantly focusing on combinatory treatments with checkpoint inhibition, are currently underway. It is well known that PD-1 or PD-L1 expression within the tumor lesion and microenvironment in general, are not sufficient surrogate markers for treatment response in patients receiving checkpoint inhibition in HCC [25]. Recently, several study groups have emphasized that mesenchymal stem cells suppress inflammation and block the powerful adaptive immune response by initiating co-inhibitory receptor expression like PD-1/PD-L1 [30, 31]. To our knowledge, there is only minor clinical evidence in large HCC cohorts to support the efficacy of immune checkpoint inhibitors in CSC-predominant HCC subtypes. It could be elucidating to investigate the PD-1 and PD-L1 status in HCC nodules harbouring homogeneous CSC-distribution and its response to checkpoint inhibitors. Eventually, patients under mono-therapy of immune checkpoint inhibition need to be co-tested for EpCAM, PD-1, and PD-L1 expression in order to obtain the predictive value towards treatment response.

Our study specifically highlighted the impact of intratumoral heterogeneity in HCC. In other gastrointestinal forms of cancer, intratumoral heterogeneity plays a major role in clinical decision-making, e.g. HER2/neu expression in gastric cancer [32]. In the study of Warneke et al., sampling procedures posed a significant risk of generating a false-negative Her2/neu status. $25 \%$ of patients with gastric cancer and Her2/neu overexpression have been neglected due to sampling errors, leading to withholding of effective treatment with trastuzumab. Additionally, a minor false-positive rate of $6 \%$ has been reported [32].

The indication to perform biopsies in HCC patients in late disease stage will increase in the future when patient distribution is required for new treatment modalities. For this reason, we addressed the issue of representative 
sampling, taking into account the EpCAM expression patterns of a single spot compared with all spots of one HCC nodule and its significance towards the EpCAM score. We found that every other patient with a positive EpCAM result from biopsy actually harbours heterogeneous EpCAM-expression, and therefore has the same prognosis of a patient with EpCAM negative HCC. In conclusion, one single biopsy does not seem to be sufficient to predict patient outcome based on EpCAMexpression. However, our results were generated from patients in early tumor stages. Hence, our conclusions cannot be transferred to stratify patients with advanced tumor stages. However, we do believe the issue of sampling errors needs to be addressed in patients in later stages, because cancers with CSC features are more resistant to systemic chemotherapy [13]. The results of the present study and its impact on clinical outcome suggest that more than one biopsy is required in order to minimize the risk of false negative or positive predictive histopathological staining.

\section{Conclusion}

CSC intratumoral heterogeneity is present in HCC nodules as demonstrated in this extensive cohort. Of note, we were able to identify one distinct HCC subgroup, which was associated with higher tumor aggressiveness, metastasesinitiation and worse clinical outcome, indicated by shorter TTR and RFS. Of note, it was characterized by homogeneous distribution of EpCAM-expression. HCC with heterogeneous EpCAM-expression had characteristics remarkably similar to HCC without any EpCAM-positive tumor cells. Especially in times when significance of tumor biopsies in late stage disease is increasing, our study highlights the risk posed by sampling errors in clinical decision-making.

\begin{abstract}
Abbreviations
AASLD: American Association for the Study of Liver Diseases; AFP: AlphaFetoprotein; AKT/mTOR: Protein Kinase B/mechanistic Target of Rapamycin; AXIN1: Axis Inhibition Protein 1; BCLC: Barcelona Clinic Liver Cancer Classification; CSC: Cancer Stem Cell; CTNNB1: Catenin Beta 1; EASL: European Association for the Study of the Liver; ECOG: Eastern Cooperative Oncology Group; EpCAM: Epithelial cell adhesion molecule; H\&E: Hematoxylin and Eosin; HBV: Hepatitis B Virus; HCC: Hepatocellular Carcinoma; HCV: Hepatitis C Virus; HER2/neu: Human Epidermal Growth Factor Receptor 2; IGF1R: InsulinLike Growth Factor 1; IHC: Immunohistochemistry; INR: International Normalized Ratio; LR: Liver resection; NASH: Non-alcoholic Steatohepatitis; PD-1: Programmed Death-1; PD-L1: Programmed Death Ligand-1; RFS: Recurrence-Free-Survival; RPS6KA3: Ribosomal Protein S6 Kinase A3; TACE: Transarterial Chemoembolization; TCF-4: Transcription factor 4; TMA: Tissue Microarray; TNM: TNM Classification of Malignant Tumors (T, tumor; N, lymph nodes; M, metastases); TP53: Tumor suppressor protein 53; TTR: Time-To-Recurrence; WNT: Wingless-Type MMTV Integration Site Family
\end{abstract}

\section{Acknowledgments}

We thank Melanie Witt and Christina Möller-Koop for excellent technical assistance. And we thank Elaine Hussey for excellent English language scientific text editing.

\section{Authors' contributions}

JK, JVF, and KS designed the study. KS, JVF, TWF, CS and HW obtained patient data. JK, GS, TK and SAW designed the TMA and conducted the staining. KS, JK and JG performed the readout of the stained TMA. CJ and HI obtained radiological follow-up data. AH, JL and LF obtained tissue specimens. HW and AWL supervised the study. CC performed all statistical analyses. JK, JVF and KS wrote the manuscript. All authors have read and approved the manuscript.

\section{Authors' information}

JK has undergone specific training for gastrointestinal and hepatobiliary pathology for 12 months supervised by GS and TK.

\section{Funding}

This work has been supported by the Deutsche Leberstiftung via a Leave Grant (KS). Open Access funding enabled and organized by Projekt DEAL.

\section{Availability of data and materials}

The datasets analysed during the current study are available from the corresponding author on reasonable request.

\section{Ethics approval and consent to participate}

The study was approved by the Ethics Committee of the Hamburg Medical Association (approval number PV-3578) and written informed consent to the study protocol was obtained from all participants prior to inclusion in this study.

\section{Consent for publication}

Please refer to the attached consent form.

\section{Competing interests}

The authors declare that they have no competing interests.

\section{Author details}

${ }^{1}$ Department of Medicine, University Medical Centre Hamburg-Eppendorf, Hamburg, Germany. ${ }^{2}$ Bioinformatics Core, University Medical Centre Hamburg-Eppendorf, Hamburg, Germany. ${ }^{3}$ Department of Diagnostic and Interventional Radiology and Nuclear Medicine, University Medical Centre Hamburg-Eppendorf, Hamburg, Germany. ${ }^{4}$ Department of Pathology, University Medical Centre Hamburg-Eppendorf, Hamburg, Germany. ${ }^{5}$ Department of General, Visceral and Thoracic Surgery, University Medical Centre Hamburg-Eppendorf, Hamburg, Germany. ${ }^{6}$ Department of Visceral Transplant Surgery, University Medical Centre Hamburg-Eppendorf, Hamburg, Germany. ${ }^{7}$ Mildred Scheel Cancer Career Centre HaTriCS4, University Medical Centre Hamburg-Eppendorf, Martinistraße 52, 20246 Hamburg, Germany.

Received: 5 July 2020 Accepted: 28 October 2020

Published online: 23 November 2020

\section{References}

1. Global Burden of Disease Cancer C, Fitzmaurice C, Akinyemiju TF, Al Lami FH, Alam T, Alizadeh-Navaei R, Allen C, Alsharif U, Alvis-Guzman N, Amini E, et al. Global, Regional, and National Cancer Incidence, Mortality, Years of Life Lost, Years Lived With Disability, and Disability-Adjusted Life-Years for 29 Cancer Groups, 1990 To 2016: A systematic analysis for the global burden of disease study. JAMA Oncol. 2018;4(11):1553-68.

2. Friemel J, Rechsteiner M, Frick L, Bohm F, Struckmann K, Egger M, Moch H, Heikenwalder M, Weber A. Intratumor heterogeneity in hepatocellular carcinoma. Clin Cancer Res. 2015;21(8):1951-61.

3. Yamashita T, Ji J, Budhu A, Forgues M, Yang W, Wang HY, Jia H, Ye Q, Qin LX, Wauthier E, et al. EpCAM-positive hepatocellular carcinoma cells are tumor-initiating cells with stem/progenitor cell features. Gastroenterology. 2009;136(3):1012-24.

4. Chan AW, Tong JH, Chan SL, Lai PB, To KF. Expression of stemness markers (CD133 and EpCAM) in prognostication of hepatocellular carcinoma. Histopathology. 2014;64(7):935-50.

5. Islam F, Gopalan V, Wahab R, Smith RA, Lam AK. Cancer stem cells in oesophageal squamous cell carcinoma: Identification, prognostic and treatment perspectives. Crit Rev Oncol Hematol. 2015;96(1):9-19.

6. Massoner P, Thomm T, Mack B, Untergasser G, Martowicz A, Bobowski K, Klocker $\mathrm{H}$, Gires $\mathrm{O}$, Puhr M. EpCAM is overexpressed in local and metastatic 
prostate cancer, suppressed by chemotherapy and modulated by METassociated miRNA-200c/205. Br J Cancer. 2014;111(5):955-64.

7. Kroepil F, Dulian A, Vallbohmer D, Geddert H, Krieg A, Vay C, Topp SA, am Esch JS, Baldus SE, Gires O, et al. High EpCAM expression is linked to proliferation and lauren classification in gastric cancer. BMC Res Notes. 2013; 6:253.

8. Ohara Y, Oda T, Sugano M, Hashimoto S, Enomoto T, Yamada K, Akashi Y, Miyamoto R, Kobayashi A, Fukunaga K, et al. Histological and prognostic importance of CD44(+)/CD24(+)/EpCAM(+) expression in clinical pancreatic cancer. Cancer Sci. 2013;104(8):1127-34.

9. Agboola AJ, Paish EC, Rakha EA, Powe DG, Macmillan RD, Ellis IO, Green AR. EpCAM expression is an indicator of recurrence in basal-like breast cancer. Breast Cancer Res Treat. 2012;133(2):575-82.

10. Yamashita T, Honda M, Nakamoto Y, Baba M, Nio K, Hara Y, Zeng SS, Hayashi T, Kondo M, Takatori H, et al. Discrete nature of EpCAM+ and CD90+ cancer stem cells in human hepatocellular carcinoma. Hepatology. 2013;57(4):1484-97.

11. Yamashita T, Forgues M, Wang W, Kim JW, Ye Q, Jia H, Budhu A, Zanetti KA, Chen Y, Qin LX, et al. EpCAM and alpha-fetoprotein expression defines novel prognostic subtypes of hepatocellular carcinoma. Cancer Res. 2008; 68(5):1451-61.

12. Xu M, Qian G, Xie F, Shi C, Yan L, Yu L, Zheng T, Wei L, Yang J. Expression of epithelial cell adhesion molecule associated with elevated ductular reactions in hepatocellar carcinoma. Clin Res Hepatol Gastroenterol. 2014; 38(6):699-705.

13. Nunes T, Hamdan D, Leboeuf C, El Bouchtaoui M, Gapihan G, Nguyen TT, Meles S, Angeli E, Ratajczak P, Lu H, et al. Targeting Cancer Stem Cells to Overcome Chemoresistance. Int J Mol Sci. 2018;19(12):4036.

14. European Association for the Study of the Liver. Electronic address eee, European Association for the Study of the L: EASL clinical practice guidelines: Management of hepatocellular carcinoma. J Hepatol. 2018;69(1): 182-236.

15. Heimbach JK, Kulik LM, Finn RS, Sirlin CB, Abecassis MM, Roberts LR, Zhu AX, Murad MH, Marrero JA. AASLD guidelines for the treatment of hepatocellular carcinoma. Hepatology. 2018;67(1):358-80.

16. Kallioniemi OP, Wagner U, Kononen J, Sauter G. Tissue microarray technology for high-throughput molecular profiling of cancer. Hum Mol Genet. 2001;10(7):657-62

17. Nocito A, Kononen J, Kallioniemi OP, Sauter G. Tissue microarrays (TMAs) for high-throughput molecular pathology research. Int J Cancer. 2001;94(1):1-5.

18. Torhorst J, Bucher C, Kononen J, Haas P, Zuber M, Kochli OR, Mross F, Dieterich $\mathrm{H}$, Moch $H$, Mihatsch $M$, et al. Tissue microarrays for rapid linking of molecular changes to clinical endpoints. Am J Pathol. 2001;159(6):224956.

19. Fong D, Seeber A, Terracciano L, Kasal A, Mazzoleni G, Lehne F, Gastl G, Spizzo G. Expression of EpCAM (MF) and EpCAM (MT) variants in human carcinomas. J Clin Pathol. 2014;67(5):408-14.

20. Zhu AX, Park JO, Ryoo BY, Yen CJ, Poon R, Pastorelli D, Blanc JF, Chung HC, Baron AD, Pfiffer TE, et al. Ramucirumab versus placebo as second-line treatment in patients with advanced hepatocellular carcinoma following first-line therapy with sorafenib (REACH): a randomised, double-blind, multicentre, phase 3 trial. Lancet Oncol. 2015;16(7):859-70.

21. Bae JS, Noh SJ, Jang KY, Park HS, Chung MJ, Park CK, Moon WS. Expression and role of epithelial cell adhesion molecule in dysplastic nodule and hepatocellular carcinoma. Int J Oncol. 2012;41(6):2150-8.

22. Marquardt JU, Andersen JB, Thorgeirsson SS. Functional and genetic deconstruction of the cellular origin in liver cancer. Nat Rev Cancer. 2015; 15(11):653-67.

23. Hayashi T, Yamashita T, Okada H, Nio K, Hara Y, Nomura Y, Hayashi T, Asahina Y, Yoshida M, Oishi N, et al. Sporadic PCDH18 somatic mutations in EpCAM-positive hepatocellular carcinoma. Cancer Cell Int. 2017;17:94.

24. Zhou L, Zhu Y. The EpCAM overexpression is associated with clinicopathological significance and prognosis in hepatocellular carcinoma patients: A systematic review and meta-analysis. Int J Surg. 2018;56:274-80.

25. Rebouissou S, Nault JC. Advances in molecular classification and precision oncology in hepatocellular carcinoma. J Hepatol. 2020;72(2):215-29.

26. Keller L, Werner S, Pantel K. Biology and clinical relevance of EpCAM. Cell Stress. 2019;3(6):165-80.

27. Schulze K, Imbeaud S, Letouze E, Alexandrov LB, Calderaro J, Rebouissou S, Couchy G, Meiller C, Shinde J, Soysouvanh F, et al. Exome sequencing of hepatocellular carcinomas identifies new mutational signatures and potential therapeutic targets. Nat Genet. 2015;47(5):505-11.

28. Schulze K, Nault JC, Villanueva A. Genetic profiling of hepatocellular carcinoma using next-generation sequencing. J Hepatol. 2016;65(5):1031-42.

29. Ho DW, Tsui YM, Sze KM, Chan LK, Cheung TT, Lee E, Sham PC, Tsui SK, Lee $\mathrm{TK}, \mathrm{Ng}$ IO. Single-cell transcriptomics reveals the landscape of intra-tumoral heterogeneity and stemness-related subpopulations in liver cancer. Cancer Lett. 2019;459:176-85.

30. Yan Z, Zhuansun Y, Liu G, Chen R, Li J, Ran P. Mesenchymal stem cells suppress $T$ cells by inducing apoptosis and through PD-1/B7-H1 interactions. Immunol Lett. 2014;162(1 Pt A):248-55.

31. Luz-Crawford P, Noel D, Fernandez X, Khoury M, Figueroa F, Carrion F, Jorgensen C, Djouad F. Mesenchymal stem cells repress Th17 molecular program through the PD-1 pathway. PLoS One. 2012;7(9):e45272.

32. Warneke VS, Behrens HM, Boger C, Becker T, Lordick F, Ebert MP, Rocken C. Her2/neu testing in gastric cancer: evaluating the risk of sampling errors. Ann Oncol. 2013;24(3):725-33.

\section{Publisher's Note}

Springer Nature remains neutral with regard to jurisdictional claims in published maps and institutional affiliations.

Ready to submit your research? Choose BMC and benefit from:

- fast, convenient online submission

- thorough peer review by experienced researchers in your field

- rapid publication on acceptance

- support for research data, including large and complex data types

- gold Open Access which fosters wider collaboration and increased citations

- maximum visibility for your research: over $100 \mathrm{M}$ website views per year

At BMC, research is always in progress.

Learn more biomedcentral.com/submissions 